\title{
The lack of effect of ejaculate sequence on sex ratio in rabbits
}

\author{
C. D’Amato, D. Hagen and P. J. Dziuk \\ Department of Animal Science, University of Illinois, \\ Urbana, Illinois 61801, U.S.A.
}

\begin{abstract}
Summary. The influence of the order of the ejaculate on the sex ratio was determined by ejaculating 1 of 5 experimental males 20 times over a period of 2 to 8 h. The sex ratio was noted for the offspring of ejaculates $1,5,10,15$ and 20 in a total of 32 litters. The sex ratio of these offspring did not deviate significantly from $50: 50$ $(P>0 \cdot 1)$ for any ejaculate in the sequence.
\end{abstract}

\section{Introduction}

The ratio of the number of males to females in animals is nearly $50: 50$ as a result of the usually accepted concept of chromosomal sex determination. The effective control of sex ratios would be desirable for those animal enterprises which could benefit from an unequal or controlled ratio. There have been many attempts to control sex ratios by various physical and biochemical methods, but the results have been equivocal (Beatty, 1970).

Frequently, there is a significant disparity from equality in observed sex ratios. The cause of this disparity is unknown (King, 1918). Dusing (1884) reported an increase in the proportion of female offspring from stallions that were mated frequently, in contrast to the proportion of offspring from stallions mated less frequently. This association may be a reflection of the order of the ejaculates occurring over a short period of time. In one widely quoted report the sex ratio in rabbits was observed to change significantly (Hayes, 1921) in litters resulting from sequential ejaculates. As the ejaculate order went from 1 to 20 the sex ratio changed from $129 \delta^{\star}: 100 q$ to $28 \sigma^{\star}: 100$. To our knowledge this observation has not been confirmed or refuted. If these observations are accurate then discovery of a possible cause perhaps would lead to a potential means for influencing sex ratio. The following experiment was designed to determine the influence that the ejaculation sequence had on the sex ratio of offspring in rabbits.

\section{Materials and Methods}

The does were New Zealand White, and the 5 bucks were New Zealand White, Dutch Belted or mixed breed. They were housed individually outdoors with food and water always available. On any one day, 1 of the 5 bucks was selected to produce 20 ejaculates in succession. Males were sexually rested for at least 12 days before use. Matings took place between 09:00 and 17:00 $\mathrm{h}$ from March to September. Five normal receptive does were assigned to be served at ejaculate numbers $1,5,10,15$ and 20 . For ejaculate numbers $2,3,4,6,7,8,9,11,12,13,14,16,17,18$ and 19 , the bucks served ovariectomized does made receptive by oestrogen. Nesting boxes were placed in the cages of pregnant does 23 days after mating. Does were observed regularly for nesting behaviour and presence of young. As soon as practical and within $48 \mathrm{~h}$ after kindling, the offspring were killed and examined under a dissecting microscope for the presence of testes or ovaries. Data on sex ratio were analysed by a $\chi^{2}$ method (see Snedecor, 1956). 


\section{Results}

When sex ratios of offspring of individual bucks were analysed no significant differences in sex ratios were observed between ejaculates $1,5,10,15$ and $20(P>0.01)$. An analysis of combined data from the 5 bucks also revealed that there was no overall effect on the sex ratio due to ejaculate order (Table 1). The mean litter size and conception rate were not affected by the ejaculate order.

Table 1. Sex ratios of rabbit offspring from successive ejaculates

\begin{tabular}{|c|c|c|c|c|c|}
\hline & \multicolumn{5}{|c|}{ Ejaculate order } \\
\hline & 1 & 5 & 10 & 15 & 20 \\
\hline No. of litters & 8 & 6 & 6 & 5 & 7 \\
\hline No. of offspring & 72 & 52 & 60 & 51 & 52 \\
\hline Conception rate (\%) & 67 & 55 & 72 & 56 & 78 \\
\hline Ratio of $\delta^{2} \partial^{2}$ to $q Q$ & $\begin{array}{c}43: 29 \\
(100: 67)\end{array}$ & $\begin{array}{c}29: 23 \\
(100: 80)\end{array}$ & $\begin{array}{c}36: 34 \\
(100: 94)\end{array}$ & $\begin{array}{c}24: 27 \\
(100: 113)\end{array}$ & $\begin{array}{c}28: 24 \\
(100: 80)\end{array}$ \\
\hline
\end{tabular}

\section{Discussion}

These results do not confirm those of the previous report (Hayes, 1921) on the influence of ejaculate order. In the present experiment the number of offspring was adequate for analysis, the conception rate and litter size were not unusual. This would indicate that fertility and embryonic losses were normal and were not likely to contribute to an undetected unequal loss of embryos from one sex before sex was noted. The fetuses were sexed at autopsy by examining for ovaries or testes under magnification thus eliminating possible errors in sexing. Hayes (1921) reported that he used the offspring in his study for subsequent studies on growth rate, and so it may be assumed, although it is not stated, that sexing was done on the basis of the appearance of the external genitalia at some unspecified time after birth. Because of the similarity in appearance of young female and male rabbits there is a possibility of error in this method which may explain the discrepancy between the results of Hayes (1921) and the present experiment.

The bucks used by Hayes were certain to be genetically different from the bucks used in the present experiment. The extent to which this genetic difference may account for the differences noted between the two experiments is unknown. The five bucks used in our experiments were of divergent genetic background but none of the individuals showed an effect of order of ejaculate and it is therefore unlikely that a possible effect of order of ejaculate is present in some rabbits and not others. In view of the present results it does not seem reasonable to search for means to understand and control sex ratios in rabbits based on the order of the ejaculate.

\section{References}

Beatty, R.A. (1970) Genetic basis for the determination of sex. Phil. trans. R. Soc. Lond. B 259, 3-14.

Dusing, K. (1884) Die regulierung der geschlechtsuerhaltnisse bei der vermehrung der menschen, tiere and pflangen. Fena. Zeit. 17, 593-642.

Hayes, F.A. (1921) Effect of excessive use of male rabbits in breeding. Exp. Zool. 25, 571-613.
King, H.D. (1918) The effect of inbreeding with selection on the sex ratio of the albino rat. J. exp. Zool. 27, 137.

Snedecor, G.W. (1956) Statistical Methods, 5th edn. Chap. 10, Iowa State College Press, Ames, Iowa.

Received 31 August 1978 